\title{
Polarization Insensitive Wavelength Conversion Based on Four-Wave Mixing in a Silicon Nanowire
}

Pu, Minhao; Hu, Hao; Peucheret, Christophe; Ji, Hua; Galili, Michael; Oxenløwe, Leif Katsuo; Jeppesen, Palle; Hvam, Jørn Märcher; Yvind, Kresten

Published in:

Advanced Photonics Congress

Publication date:

2012

Document Version

Publisher's PDF, also known as Version of record

Link back to DTU Orbit

Citation (APA):

Pu, M., Hu, H., Peucheret, C., Ji, H., Galili, M., Oxenløwe, L. K., Jeppesen, P., Hvam, J. M., \& Yvind, K. (2012). Polarization Insensitive Wavelength Conversion Based on Four-Wave Mixing in a Silicon Nanowire. In Advanced Photonics Congress (pp. IW4C.4). Optical Society of America.

\section{General rights}

Copyright and moral rights for the publications made accessible in the public portal are retained by the authors and/or other copyright owners and it is a condition of accessing publications that users recognise and abide by the legal requirements associated with these rights.

- Users may download and print one copy of any publication from the public portal for the purpose of private study or research.

- You may not further distribute the material or use it for any profit-making activity or commercial gain

- You may freely distribute the URL identifying the publication in the public portal 


\title{
Polarization Insensitive Wavelength Conversion Based on Four-Wave Mixing in a Silicon Nanowire
}

\author{
M. Pu, H. Hu, C. Peucheret, H. Ji, M. Galili, L. K. Oxenløwe, P. Jeppesen, J. M. Hvam, and K. Yvind \\ DTU Fotonik, Department of Photonics Engineering, Technical University of Denmark, Building 343, DK-2800 Lyngby, Denmark \\ mipu@fotonik.dtu.dk
}

\begin{abstract}
We experimentally demonstrate, for the first time, polarization-insensitive wavelength conversion of a $10 \mathrm{~Gb} / \mathrm{s}$ NRZ-OOK data signal based on four-wave mixing in a silicon nanowire with bit-error rate measurements.

OCIS codes: (190.4380) Nonlinear optics, four-wave mixing; (190.4390) Nonlinear optics, integrated optics; (230.7370)

Waveguide; (130.7405) Wavelength conversion devices.
\end{abstract}

\section{Introduction}

All-optical wavelength conversion (AOWC) is an important feature in future wavelength division multiplexing (WDM) networks. This functionality has been demonstrated in different devices including semiconductor optical amplifiers (SOAs) [1], periodically-poled lithium niobate (PPLN) waveguides [2], and highly nonlinear fibers (HNLFs) [3]-[6], based on different nonlinear effects such as cross-phase modulation (XPM), cascaded second harmonic and difference frequency generation (cSHG/DFG), and four-wave-mixing (FWM). Recently, nonlinear effects in silicon nanowires have attracted considerable research interests due to compactness, large conversion bandwidth and complementary metal-oxide-semiconductor (CMOS) compatibility. Due to the strong light confinement in silicon waveguides with sub-micron dimensions, the group velocity dispersion (GVD), which is a critical parameter for parametric processes, can be engineered and thus one can achieve ultra-broadband wavelength conversion [7], [8]. Previously, different nonlinear applications including signal regeneration [9], parametric amplification [10], wavelength conversion [11], [12], ultra-fast waveform sampling, demultiplexing [13], and multicasting [14] have been demonstrated based on FWM processes in silicon nanowires. The FWM process is normally highly polarization dependent and an efficient FWM process occurs when the input signal and pump waves are both aligned to either transverse-electric (TE) mode or transverse-magnetic (TM) mode. However, a polarization insensitive operation is desired since the state of polarization (SOP) of the input signal fluctuates with time and distance in a real transmission system. Various polarization-insensitive FWM-based AOWC techniques have been demonstrated in HNLFs such as polarization diversity [3], co-polarized-pump [4], orthogonal-pump [5], and $45^{\circ}$-pump schemes [6]. However, such a technique has not yet been demonstrated in silicon nanowires.

In this paper, we report polarization insensitive AOWC of a 10-Gb/s non-return-to-zero on-off keying (NRZOOK) data signal based on FWM in a silicon nanowire. Error-free performance is achieved for the converted signals while the polarization of the input data signal is continuously scrambled.

\section{Principle of operation}
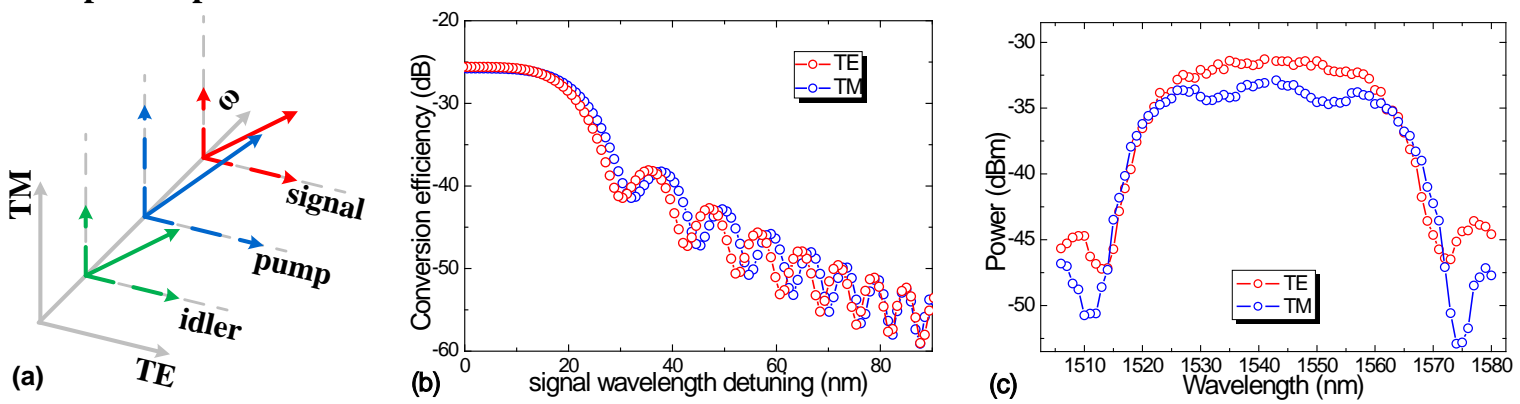

Fig. 1. (a) Schematic of the angled-pump four-wave mixing. (b) Simulated conversion efficiency versus signal wavelength detuning from the pump $(1542 \mathrm{~nm})$ for the TE and TM modes for the silicon nanowire $\left(300 \times 450 \mathrm{~nm}^{2}\right)$. (c) Measured conversion efficiency versus input signal wavelength with a fixed input pump at wavelength $1542 \mathrm{~nm}$ for the silicon nanowire $\left(300 \times 450 \mathrm{~nm}^{2}\right)$.

FWM is intrinsically a highly polarization dependent process. Usually, the pump and the signal waves have to be copolarized and both aligned to one of the fundamental waveguide modes (TE or TM modes) to get an efficient FWM process in a silicon nanowire. However, if the SOP of the pump wave is intentionally set at a certain angle so that it couples to both the TE and TM modes of the waveguide, the scheme can be polarization insensitive [15] as shown in Fig. 1 (a). In a silicon nanowire, the angled pump will be split into two orthogonal polarization components. If the pump polarization angle is carefully adjusted to make the power of the projections of the input pump field along the 
TE and TM axes just counterbalance the difference in conversion efficiencies between the TE and TM modes, then the conversion efficiency will be the same for signals aligned to the TE or TM axes, hence polarization-independent. For instance, if the conversion efficiency of the TE mode is higher than that of the TM mode, then the angle of the pump is adjusted so that lower pump power is coupled to the TE mode than to the TM mode. As a result, the output converted signal (idler wave) which is the sum of its TE and TM modes components, will keep constant regardless of the SOP of the input signal wave. The resultant polarization insensitive conversion efficiency and bandwidth are then mainly limited by the TE or TM mode that has the lower conversion efficiency and narrower bandwidth. Therefore, it is important to design a silicon nanowire with similar conversion performances for both the TE and TM modes to realize an efficient polarization-insensitive wavelength conversion. Although a typical silicon nanowire has different dispersion properties for the different polarization modes due to the structure asymmetry, its dispersion can be carefully engineered by tailoring the waveguide geometry to obtain similar conversion performances for the TE and TM modes when pumping at a certain wavelength.

The silicon nanowire used in this work is 1 -cm long and its cross-sectional dimension $\left(\mathrm{H} \times \mathrm{W}=300 \times 450 \mathrm{~nm}^{2}\right)$ is engineered to obtain similar FWM performances for both polarizations. It was fabricated on silicon-on-insulator (SOI) material using electron-beam lithography followed by reactive-ion etching. The silicon nanowire was inversely tapered at both ends and covered by a polymer waveguide for efficient coupling [16]. Fig. 2(b) shows the simulated conversion efficiencies for the TE and TM modes as a function of signal wavelength detuning from the pump (at $1542 \mathrm{~nm}$ ). It is observed that the conversion bandwidths for both modes are very similar since their dispersion has the same magnitude (but opposite sign) at the pump wavelength. Fig. 1(c) shows the measured conversion efficiency as a function of signal wavelength when the pump and signal are both aligned with the TE and TM modes. In the measurement, the pump wavelength and power are $1542 \mathrm{~nm}$ and $17.9 \mathrm{dBm}$, respectively. The conversion efficiencies for the TE and TM mode are $-31.2 \mathrm{~dB}$ and $-32.9 \mathrm{~dB}$, respectively. The 3-dB conversion bandwidths for the TE and TM modes are $35 \mathrm{~nm}$ and $43 \mathrm{~nm}$, respectively. The small efficiency difference is partly due to the different fiber-to-chip coupling and propagation losses for the different waveguide modes.

\section{Experiment}

The experimental setup for polarization-insensitive AOWC of a 10-Gb/s NRZ-OOK signal is shown in Fig. 2. The pump wave is generated from an external cavity laser $\left(E C L_{1}\right)$ at $1542 \mathrm{~nm}$. The signal wave, generated by $\mathrm{ECL}_{2}$ at $1550 \mathrm{~nm}$, is externally modulated by a Mach-Zehnder modulator (MZM) with a 10-Gb/s NRZ-OOK signal, encoded with a pseudo-random binary sequence (PRBS) of $2^{31}-1$. The SOP of the signal is continuously scrambled over the entire Poincaré sphere with a frequency of the order of $700 \mathrm{kHz}-1 \mathrm{MHz}$. Both signal and pump are amplified by erbium-doped fiber amplifiers (EDFAs), filtered by 1-nm optical band-pass filters (OBPFs), and then combined in a 3-dB coupler. Two tapered fibers are used for coupling light into and out of the silicon nanowire. The polarization controller (PC) is used to adjust the polarization angle for the pump. At the output of the silicon nanowire, the converted signal (idler wave) is filtered out by two OBPFs (the first is a 3-nm Gaussian-shape OBPF and the second a 1-nm rectangular-shape OBPF), amplified by an EDFA in between, and detected by the 10-Gb/s OOK receiver (shown by the dashed box in Fig. 2). In the receiver, the 10-Gb/s NRZ-OOK data signal is pre-amplified, filtered with a 1-nm OBPF and then detected by a photodetector (PD), followed by a 10-Gb/s error analyzer (EA) for BER measurements. An optical spectrum analyzer (OSA) is used to measure the output spectrum.

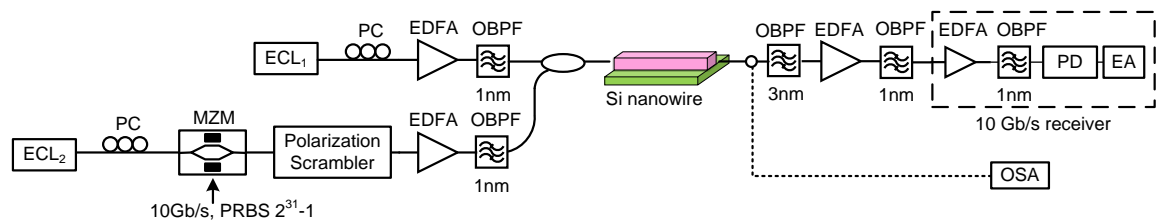

Fig. 2. Experimental setup for polarization insensitive wavelength conversion of $10 \mathrm{~Gb} / \mathrm{s}$ NRZ-OOK data signal in a silicon nanowire.

Fig. 3 (a) shows the spectrum measured at the output of the silicon nanowire when signal polarization scrambling is used. The CW pump, signal and idler waves are denoted $\lambda_{\mathrm{p}}, \lambda_{\mathrm{s}}$, and $\lambda_{\mathrm{i}}$, respectively. The input powers of the pump and signal are $17.9 \mathrm{dBm}$ and $9.5 \mathrm{dBm}$, respectively. The conversion efficiency of the generated idler is $-40.6 \mathrm{~dB}$. The polarization of the pump wave was adjusted to minimize the signal distortion at the receiver while the polarization of the input signal was being scrambled. The lower conversion efficiency than the one shown in Fig. 1(c) is due to the about 3-dB lower pump power coupled to both TM or TE modes as a result of the pump field being projected nearly equally onto the two polarization modes.

To evaluate the performance of the converted data signal and the polarization insensitivity of the AOWC, we measured the BER for the $10 \mathrm{~Gb} / \mathrm{s}$ NRZ-OOK back-to-back and the converted data signals without and with the signal polarization scrambling. The signal polarization is in a random state when its polarization is not scrambled. 
As shown in Fig. 3 (b), error-free operation without an error floor was achieved for the converted signal in both cases. The power penalty for the wavelength converted signal is around $3.7 \mathrm{~dB}$ at the BER of $10^{-9}$ compared with the back-to-back case, which is mainly due to the residual CW pump and the relatively low optical signal-to-noise ratio (OSNR) of the converted signal. The receiver sensitivity for the converted signal could be improved by better filtering away the residual CW pump or increasing the pump power, which would improve the conversion efficiency and result in an increased OSNR. The additional power penalty caused by the polarization scrambling is smaller than $1 \mathrm{~dB}$. Figs. 3 (c, d) show eye diagrams of the 10-Gb/s NRZ-OOK data signals without and with input signal polarization scrambling, respectively. It is seen that the wavelength converted signals have clear and open eyes in both cases.
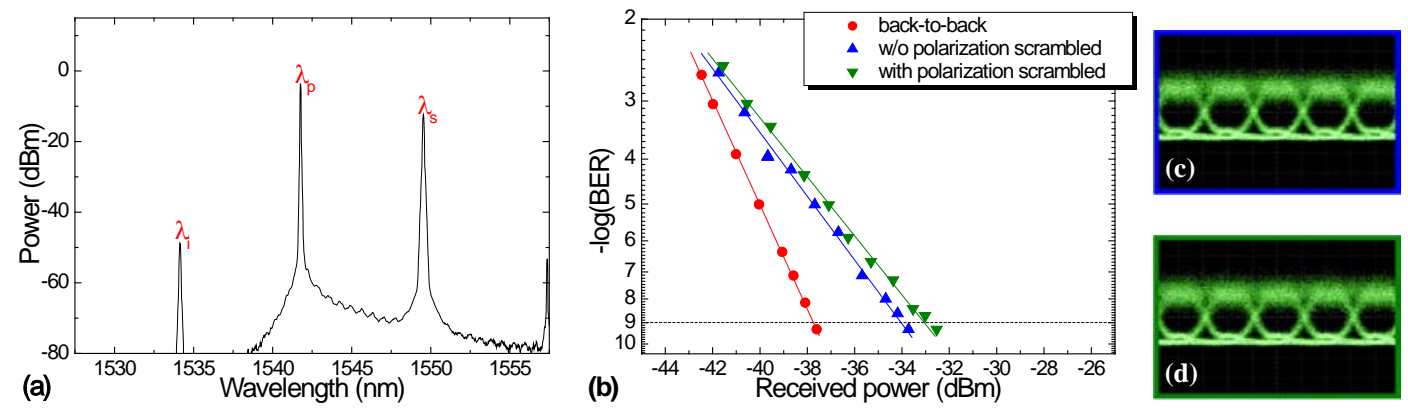

Fig. 3. (a) Measured optical spectrum at the output of the silicon nanowire. (b) BER measurement for the $10 \mathrm{~Gb} / \mathrm{s}$ NRZ-OOK back-to-back signal and the converted signal without and with the input signal being polarization-scrambled. Measured eye diagrams for the converted signal without (c) and with (d) the input signal being polarization-scrambled.

\section{Conclusion}

We have experimentally demonstrated polarization-insensitive all-optical wavelength conversion of a 10-Gb/s NRZOOK data signal. The polarization-insensitive operation is based on angled-pump FWM in a 1-cm long dispersion engineered silicon nanowire. Error-free performance is achieved for the wavelength converted signal regardless of whether the signal polarization scrambling is on or off. The residual polarization sensitivity is smaller than $1 \mathrm{~dB}$.

\section{Reference}

[1] C. M. Gallep, O. Raz, and H. J.S. Dorren, "Polarization Independent Dual Wavelength Converter Based on FWM in a Single Semiconductor Optical Amplifier,” in Proc. OFC 2010, paper OWP2 (2010).

[2] H. Hu, R. Nouroozi, R. Ludwig, B. Huettl, C. Schmidt-Langhorst, H. Suche, W. Sohler and C. Schubert, "Polarization-insensitive alloptical wavelength conversion of 320 Gb/s RZ-DQPSK signals using a Ti:PPLN waveguide,” Applied Physics B 101, 875-882 (2010).

[3] H. Hu, E. Palushani, M. Galili, H. C. H. Mulvad, A. Clausen, L. K. Oxenløwe, and P. Jeppesen, "640 Gbit/s and 1.28 Tbit/s polarisation insensitive all optical wavelength conversion,” Opt. Express 18(10), 9961-9966 (2010).

[4] J. Ma, J. Yu, C. Yu, Z. Jia, X. Sang, Z. Zhou, T. Wang, and G. K. Chang, "Wavelength conversion based on four-wave mixing in highnonlinear dispersion shifted fiber using a dual-pump configuration,” IEEE J. Lightwave Technol. 24(7), 2851-2858 (2006).

[5] K. Inoue, "Polarization independent wavelength conversion using fiber four-wave mixing with two orthogonal pump lights of different frequencies,” J. Lightwave Technol. 12(11), 1916-1920 (1994).

[6] F. Yaman, Q. Lin, and G. P. Agrawal, “A novel design for polarization-independent single-pump fiber-optic parametric amplifiers,” IEEE Photon. Technol. Lett., 18(22), 2335-2337 (2006).

[7] M. A. Foster, A. C. Turner, R. Salem, M. Lipson and A. L. Gaeta, "Broad-band continuous-wave parametric wavelength conversion in silicon nanowaveguides,” Opt. Express 15(20), 12949 (2007).

[8] M. Pu, H. Hu, M. Galili, H. Ji, L.K. Oxenløwe, K. Yvind, P. Jeppsen, and J.M. Hvam, “15 THz tunable wavelength conversion of picosecond pulses in silicon waveguide,” IEEE Photon. Technol. Lett. 23(19), 1409-1411 (2011).

[9] R. Salem, M. A. Foster, A. C. Turner, D. F. Geraghty, M. Lipson, and A. L. Gaeta, "Signal regeneration using low power four-wave mixing on silicon chip," Nat. Photonics 2(1), 35-38 (2008).

[10] M. A. Foster, A. C. Turner, J. E. Sharping, B. S. Schmidt, M. Lipson, and A. L. Gaeta, "Broad-band optical parametric gain on a silicon photonic chip,” Nature 441(7096), 960-963 (2006).

[11] H. Rong, Y. H. Kuo, A. Liu and M. Paniccia, and O. Cohen, "High efficiency wavelength conversion of $10 \mathrm{~Gb} / \mathrm{s}$ data in silicon waveguides," Opt. Express 14(3), 1182-1188 (2006).

[12] H. Hu, H. Ji, M. Galili, M. Pu H. C. H. Mulvad, L. K. Oxenløwe, K. Yvind, J. M. Hvam, and P. Jeppesen, "Ultra-high-speed wavelength conversion in a silicon photonic chip,” Opt. Express, 19(21), 19886-19894 (2011).

[13] H. Ji, M. Pu, H. Hu, M. Galili, L.K. Oxenløwe, K. Yvind, J.M. Hvam, and P. Jeppsen, "Optical Waveform Sampling and Error-free Demultiplexing of 1.28 Tbit/s Serial Data in a Nano-engineered Silicon Waveguide,” J. Lightwave Technol. 29(4), $426-431$ (2011).

[14] M. Pu, H. Hu, M. Galili, H. Ji, L.K. Oxenløwe, K. Yvind, P. Jeppsen, and J.M. Hvam, "Non-Degenerate Four-Wave Mixing in a Silicon Nanowire and its Application for One-to-Six WDM Multicasting,” in Proc. ECOC 2011, paper Th.11.LeSaleve.2 (2011).

[15] S. Gao, X. Zhang, Z. Li, and S. He, "Polarization-Independent Wavelength Conversion Using an Angled-Polarization Pump in a Silicon Nanowire Waveguide,”IEEE J. Sel. Topics Quantum Electron. 16(1), 250-256 (2010).

[16] M. Pu, L. Liu, H. Ou, K. Yvind, and J. M. Hvam, “Ultra-low-loss inverted taper coupler for silicon-on-insulator ridge waveguide,” Opt. Commun. 283, 3678-3682 (2010). 